\title{
Multiband Three-Layer GNSS Microstrip Antenna
}

\author{
Evgeniy R. Gafarov, Alexey A. Erokhin, Andrey V. Stankovsky, Yuri P. Salomatov \\ Institute of Engineering Physics and Radio Electronics \\ Siberian Federal University \\ Krasnoyarsk, Russia \\ EGafarov@sfu-kras.ru
}

\begin{abstract}
There are various receiving antennas of satellite radio navigation systems. As a rule, the qualitative polarization characteristics (axial ratio) are achieved by efficiency degradation or frequency ranges decreasing. In this article, a circular polarization GNSS antenna, which have no disadvantages described above are presented. By using a new planar feeding network and a three layer design of patch elements, it is possible to provide a symmetrical radiation pattern with high axial ratio and efficiency in various GNSS frequency bands L1/L2/L5/G1/G2/G3/E1/E2/E5ab//B1/B2 et al. Moreover, in the proposed antenna the capacitive caps were used to feed microstrip patch. It allowed getting good reflection coefficient in the extended frequency range $(\sim 10 \%$ for one patch element) and receiving of all GNSS signals by just two resonant elements. In the article, the breadboarding was carried out and the experimental results of electrodynamic characteristics were obtained. Perspective application of the proposed antenna design is demonstrated using these characteristics. It should be noted that the proposed antenna has low weight and low manufacturing cost due to the use of microstrip technology.
\end{abstract}

Index Terms - GNSS, antenna, circular polarization.

\section{INTRODUCTION}

There are plurality of antennas for receiving radio navigation signal at multiple frequencies (L1, L2, L5 GPS etc.). The different types of global navigation satellite system (GNSS) antennas are characterized by various parameters. For example, spiral slot antenna [1] has an excellent polarization characteristics and stable phase center. However, the disadvantage of such an antenna is low efficiency. The microstrip patch antenna also is used in the field of satellite radio navigation systems [2]. The advantage of microstrip antenna with comparison the spiral slot antenna is a high efficiency at the resonance frequency. However, to ensure high axial ratio [3] it is necessary to use several feeding points of the resonant patch with a constant phase shift of each output as it is shown in [4] for single-frequency mode. However, the number of feeding points could not be too large. Otherwise due to the transferring of power between the ports the efficiency of the antenna will be decreased. To provide the dual-band mode, it is necessary to use two radiating patch and broadband divider with a phase shift between the outputs [5]. The implementation of such requirements to the patch antenna can proposes the newest antenna for receiving navigation signals.
Further multiband three-layer patch antenna with high efficiency and high axial ratio will be presented.

\section{ANTENNA CONFIGURATION}

To obtain an antenna operating at high (L1, G1 and etc.) and low (L2, G2 and etc.) frequencies with high efficiency and AR the design of a multilayer resonant patch antenna was investigated. Moreover, a planar broadband power splitter was designed to feed power at several points to a patch.

\section{A. Number of feeding points}

Depending on the number of feeding points the different polarization characteristics and efficiency can be obtained. Next, we consider three options of the same amplitude but different phase between points, i.e.:

- two feeding points with phases $0^{\circ}, 90^{\circ}$;

- $\quad$ three feeding points with phases $0^{\circ}, 120^{\circ}, 240^{\circ}$;

- four feeding points with phases $0^{\circ}, 90^{\circ}, 180^{\circ}, 270^{\circ}$.

The antenna efficiency for three power points is the sum of the insertion loss in splitter substrate $(\sim 3 \%)$ and return loss (transfer of power between port 1 and the other two $(2,3)$ Fig. 1a). In other words, the transmission coefficient between feeding points makes a major contribution to reducing efficiency. In the case of three feeding points $S_{21}=S_{31}$ and is $12 \mathrm{~dB}$ or less. The efficiency of an antenna with three feeding points is more than $92 \%$.

When we use four feeding points (Fig. 1c) $\mathrm{S}_{31}=-3.5 \mathrm{~dB}, \mathrm{~S}_{21}$ $=\mathrm{S}_{41}=-16 \mathrm{~dB}$ and below. Structure of four feeding points demonstrates the best circular polarization of showed circuits.

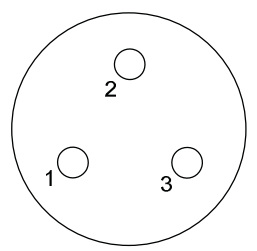

a

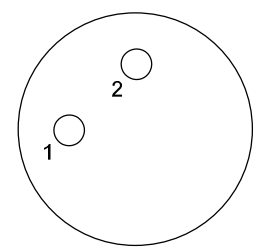

b

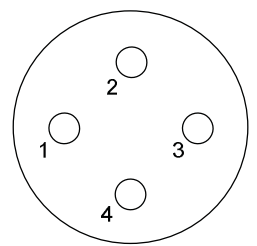

$\mathrm{c}$
Fig. 1. Feed network of three (a), two (b), four (c) feeding points 


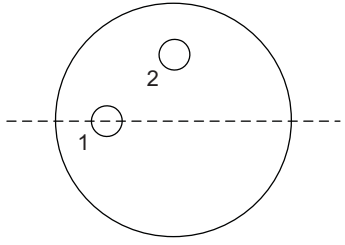

Fig. 2. Asymmetry of patch for exciting port 1

However, due to the large value of the transmission coefficient $\left(\mathrm{S}_{31}=-3.5 \mathrm{~dB}\right)$ to feeding point on the same plane, a significant degradation of antenna efficiency will be observed (about 50-60\%).

The smallest transmission coefficient between the ports $\mathrm{S}_{21}$ $=-15 \mathrm{~dB}$ is achieved when using two power points (Fig. 1b). Consequently it will lead to a better efficiency than case of three feeding points by $2-3 \%$. However, the patch antenna with two feeding points has the smallest AR of circuits presented here. Because of the asymmetry in the patch geometry for the excited port 1 (Fig. 2), a deviation of the maximum of radiation pattern is occurs. For port 2 deviations are the same in other orthogonal plane. It results in a distortion of the radiation pattern and axial ratio.

The considered circuits of feeding points allow concluding that the three-point mode is the best. The three point mode take advantages of the 2 and 4 feeding points on the other hand their disadvantages were eliminated.

\section{B. Radiating elements}

The two-layer patch antenna is used to cover the lowfrequency (L2, G2, E2 et al) and high-frequency (L1, G1, E1 et al) ranges of the radio navigation signals. To extend the bandwidth of the subrange (for example high frequency range), the capacitive caps under the high frequency patch is used. The caps are connected to splitter via holes in the lowfrequency patch (Fig. 4.). The best way to realize caps is using a dielectric substrate, which on one side there is patch, on the other side there are capacitive caps to provide electromagnetic coupling. The Rogers RO4003C dielectric substrate was used for the high-frequency patch. The low frequency patch is used for radiation at a low frequency. The low frequency patch element is realized by galvanized steel and it has polygon shape for easiest manufacture.

\section{Power splitter}

The splitter consists of quarter-wave line segments with unequal wave impedance and Wilkinson power divider. Fig. 3 shows bottom view of presented antenna, where splitter is located. At first, the power is divided by three outputs with equal amplitudes by Wilkinson divider and then there is a phase shifts between the outputs in a wide frequency band by proposed splitter. The splitter is located under the screen of the antenna. Proposed splitter is realized on a dielectric substrate Rogers RO 4003 with permittivity 3.55. On the same substrate high frequency patch is developed.

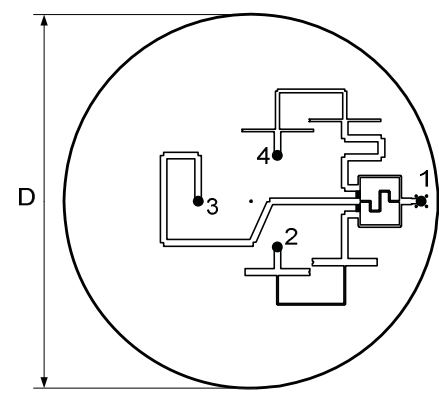

Fig. 3. Geometry of the proposed power splitter.

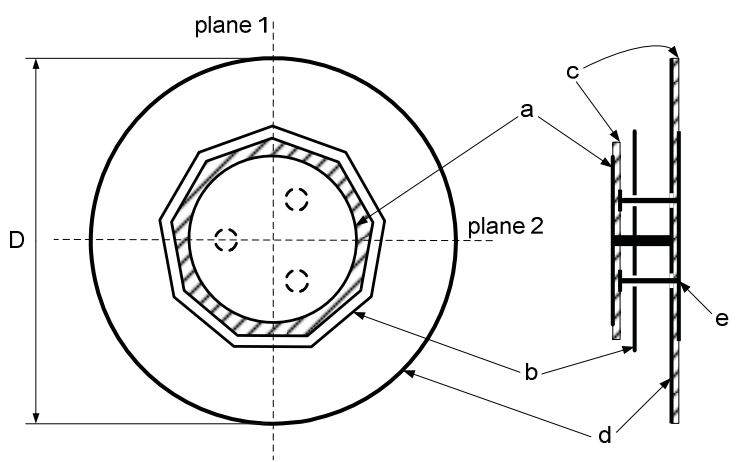

Fig. 4. Geometry of the proposed antenna: $a-$ high frequency patch; $b-$ low frequency patch; $\mathrm{c}$ - substrate; $\mathrm{d}$ - ground plane; $\mathrm{e}$ - power splitter

\section{Assembly design of antenna}

The CAD model of multiband three-layer patch antenna was developed (Fig. 4) and then breadboarding was constructed. The antenna consists of a power splitter with three equal amplitude outputs with a phase difference $\left(0^{\circ}\right.$, $120^{\circ}, 240^{\circ}$ ) on outputs and low frequency (LF) and high frequency $(\mathrm{HF})$ patches.

To install patches on ground plane a metal rod positioned in the geometric center of the antenna is used. The diameter of the HF patch is $88.5 \mathrm{~mm}$; LF patch size is $124 \mathrm{~mm}$. The diameter of the ground plane $\mathrm{D}$ is $180 \mathrm{~mm}$. The thickness of splitter substrate and HF patch substrate is the same (1.52 $\mathrm{mm})$. The thickness of LF patch on air substrate is $0.5 \mathrm{~mm}$. The output power from splitter is supplied by means of metal pins on the capacitive caps, which have no electrical contact with the patch.

\section{FIELD ANALYSIS}

As described above, at first the theoretical study was carried out and modelling of the proposed three layer antenna was performed. Then, breadboarding was made and electrodynamic characteristics were measured in an anechoic chamber. The article presents the computer modelling results only for the power splitter, because it was not possible to carry out an experimental study of the splitter. In the other hand the modelling results of assembly antenna are not presented in the article. However, it should be noted that the results of the model and the measurement are similar. 


\section{A. Modelling of power splitter}

The three outputs broadband splitter was developed by math-modeling techniques. The splitter is characterized by equal signal amplitude and a phase difference $0^{\circ}, 120^{\circ}$ and $240^{\circ}$ at the range of navigation frequencies. By means of electrodynamic modeling optimizer, the good results were achieved. Fig. 5 shows the frequency response, the input and output ports are presented in Fig. 3. From the frequency dependence of the reflection coefficient we can see that the structure of the splitter has resonant behavior, and it is calculated to the average frequency of the navigation bands $1.4 \mathrm{GHz}$. However, at $1.6 \mathrm{GHz}$ and $1.2 \mathrm{GHz}$, the reflection coefficient $\mathrm{S}_{11}$ is more than $-15 \mathrm{~dB}$. The dispersion of transmission coefficients $S_{21}, S_{31}, S_{41}$ at navigation frequencies is not more than $0.5 \mathrm{~dB}$.

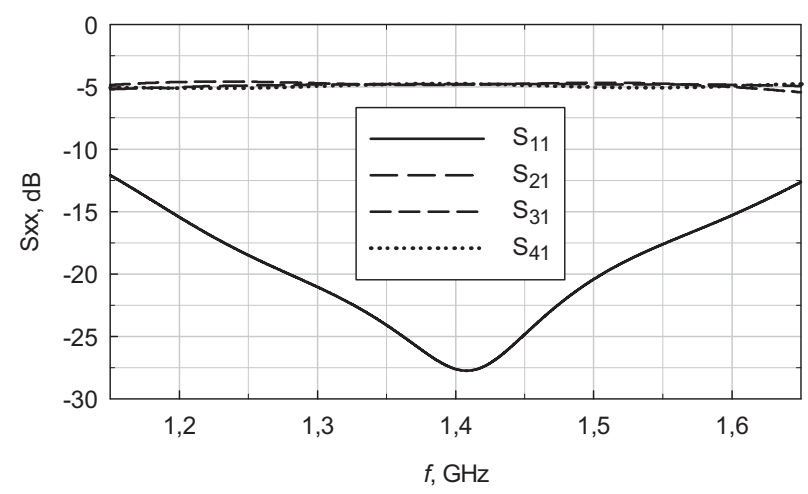

Fig. 5. Amplitude response of proposed power splitter.

The phase response is shown in Fig. 6. The phase deviation from $120^{\circ}$ between ports is less than $2.5^{\circ}$.

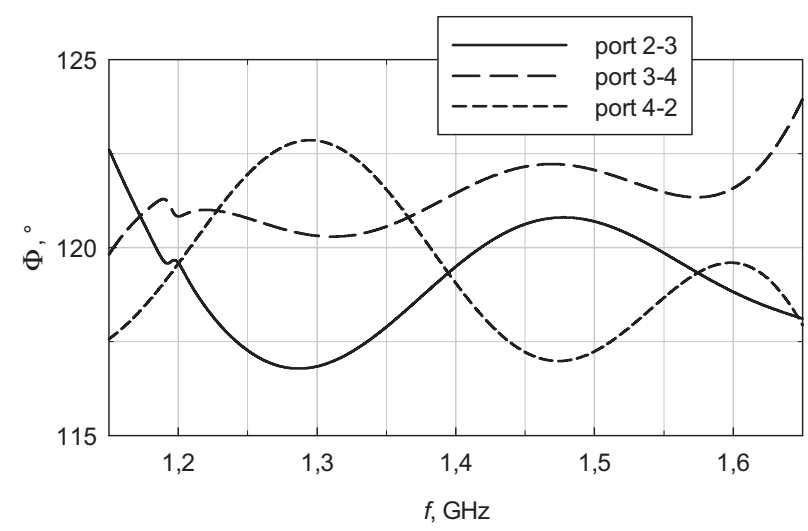

Fig. 6. Phase response of proposed power splitter.

The main advantage of the developed splitter is a planar single layer realization by microstrip technology only. In addition, the splitter does not has discrete components like phase shifter.

\section{B. Measurements of antenna}

The constructed antenna was measured in an anechoic chamber. It is shown in Fig. 7.
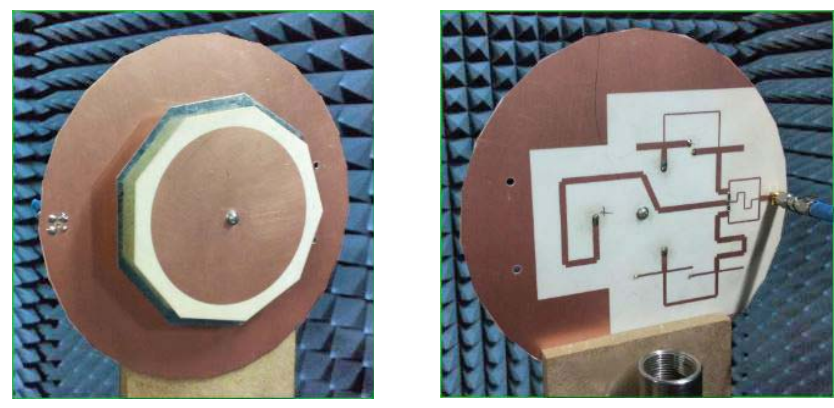

Fig. 7. Photo of proposed antenna in anechoic chamber.

The far-field radiation patterns for right hand circular and cross-polarization, as well as antenna gain were measured. Radiation patterns were measured at two antenna planes, as it is shown in Fig. 4 (plane 1 and plane 2). Radiation patterns were obtained by measuring the amplitude and phase of the transmission coefficient for two orthogonal linear polarizations. Then, using a math post processing, the radiation patterns for the right and cross polarization were calculated [6]. The measured radiation patterns are presented in Fig. 8-10 at frequencies: 1.6 GHz, $1.245 \mathrm{GHz}, 1.2 \mathrm{GHz}$, respectively.

As seen from figures presented below, the antenna has radiation pattern width is about $60^{\circ}$ equal as well $\mathrm{HF}$ as LF. The realized gain is more than $9 \mathrm{~dB}$ (Fig. 11). High AR and low cross-polarization level (less than $-17 \mathrm{~dB}$ ) in the reference direction was obtained. At the angles up to 90 degrees the antenna has a high level of cross-polarization. It is typically to this kind of radiating element. The reflection coefficient of the antenna is less than $-15 \mathrm{~dB}$.
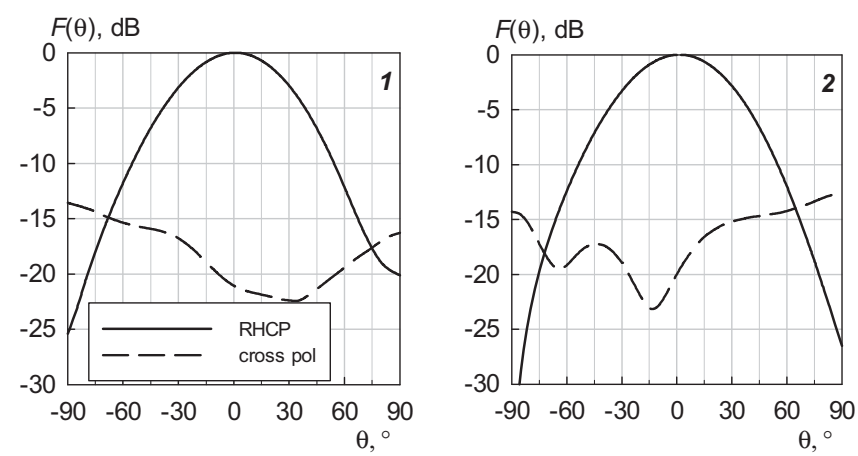

Fig. 8. Measured radiation patterns for right (RHCP) and left (cross pol) polarization at $1.6 \mathrm{GHz}$ in vertical (1) and horizontal (2) planes. 

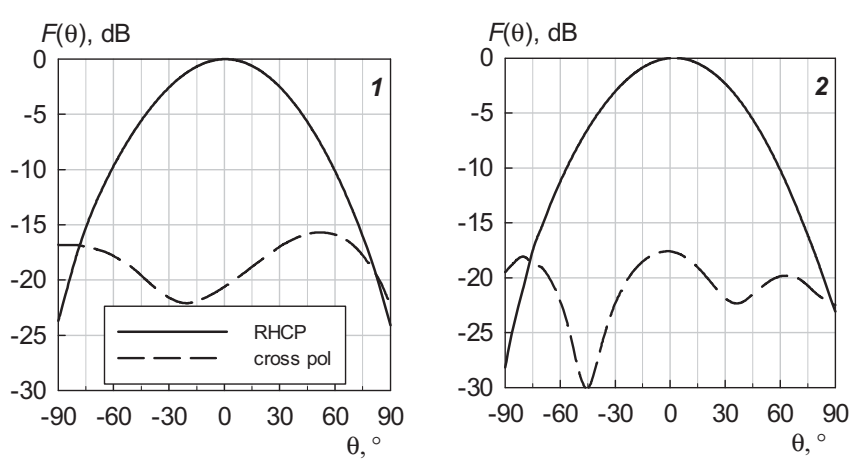

Fig. 9. Measured radiation patterns for right (RHCP) and left (cross pol) polarization at $1.245 \mathrm{GHz}$ in vertical (1) and horizontal (2) planes.
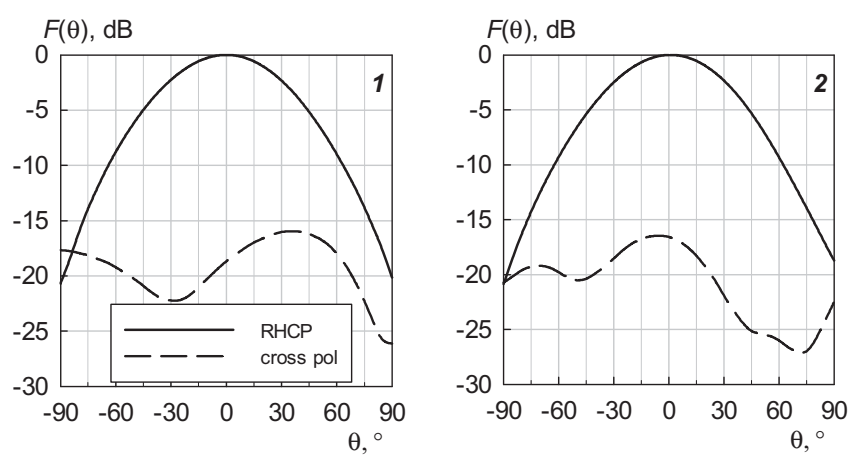

Fig. 10. Measured radiation patterns for right (RHCP) and left (cross pol) polarization at $1.2 \mathrm{GHz}$ in vertical (1) and horizontal (2) planes.

Fig. 11 and Fig. 12 show the Gain and efficiency versus frequency of the proposed antenna. From the characteristics it should be noted, that despite on the wideband properties of the splitter the resonant patch elements are operating at high and low frequencies. The behavior of resonant patches provides selectivity at out of band frequencies. In other words, the antenna does not receive signals out of operating frequency bands and it is protected from out-of-band interference.

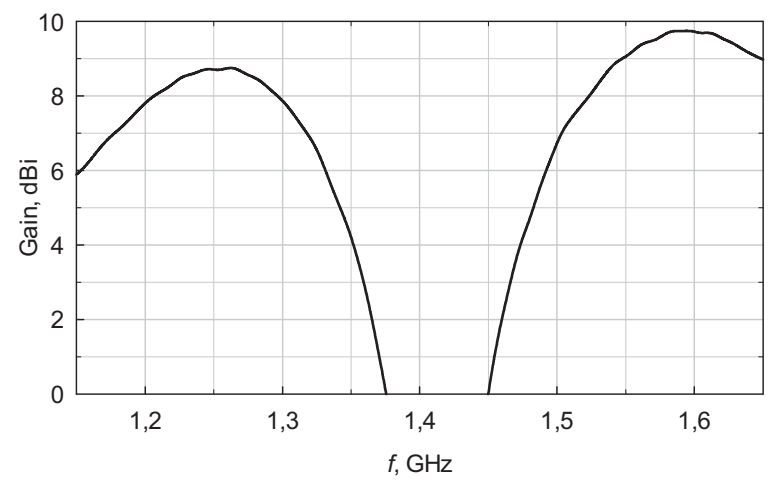

Fig. 11. Gain versus frequency of proposed antenna.

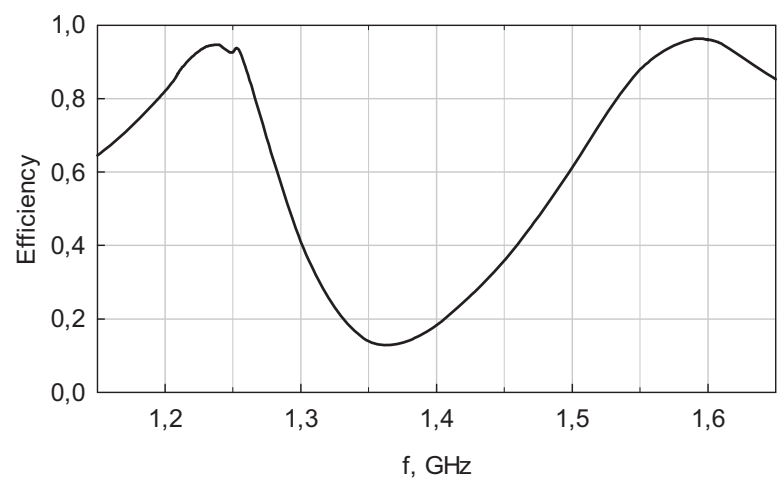

Fig. 12. Gain versus frequency of proposed antenna.

It should be noted that, unlike many solutions existing today, the antenna proposed in the article has a symmetric radiation patterns, excellent characteristics of the right circular polarization and high efficiency.

\section{CONCLUSION}

Modeling and experimental study of a multiband two-layer patch antenna was described. A feature of proposed antenna is using of three feeding points of patch and broadband feeding network. To provide operation at high and low frequencies with three points the high AR (as in the four feeding points mode) and high efficiency (as an antenna with two feeding points) can be obtained. Using capacitive caps feeding of patch allows ensuring reception of all existing navigation signals. The investigation and analysis of the results shows that the antenna operates in dual-bands (HF and LF) with right circularly polarization and efficiency more than $90 \%$. Thus, the proposed antenna can be used for signals of global navigation satellite systems.

\section{REFERENCES}

[1] V.N. Shepov, V.M. Vladimirov, V.V. Markov, "The slot stripline antenna with a jump in the slot radiators width for high accuracy GLONASS/GPS positioning," IEEE Microwave \& Telecommunication Technology (CriMiCo), 25th International Crimean Conference, Sevastopol, pp. 431-432, 2015.

[2] C.M.Su, K.L.Wong, "A dual-band GPS microstrip antenna," Microw. Opt. Technol. Lett., vol. 33, no. 4, pp. 238-240, May 2002.

[3] E. R. Gafarov, Y. P. Salomatov, "Hexagonal FSS for GLONASS/GPS antenna with improved axial ratio," International Siberian Conference on Control and Communications. pp. 159-161, September 2011. DOI: 10.1109/SIBCON.2011.6072620.

[4] Chen Lin, Fu-Shun Zhan, "A Three-Fed Microstrip Antenna for Wideband Circular Polarization," Antennas and wireless propagation letters, vol. 9, pp. 359-362, 2010. DOI: 10.1109/LAWP.2010.2048296.

[5] Du Li, G. Pengfei, D. Qing, Fu. Yunqi, "Broadband Capacitively Coupled Stacked Patch Antenna for GNSS Applications," IEEE Antennas and wireless propagation letters, Vol. 11, 2012. DOI: 10.1109/LAWP.2012.2205129.

[6] K. V. Lemberg, Y. P. Salomatov, E. R. Gafarov, "GNSS antenna characteristics using bi-orthogonal far-field measurement," IEEE Microwave \& Telecommunication Technology (CriMiCo), 24th International Crimean Conference, Sevastopol, pp. 932-933, September. 2014. DOI: 10.1109/CRMICO.2014.6959700. 